${ }^{1}$ Федеральное
государственное

бюджсетное учреждение «Российский научный центр радиологии и хирургических технологий имени академика

\section{А.М. Гранова»}

Министерства здравоохранения Российской Федерации (Санкт-Петербург, Россия)

${ }^{2}$ Санкт-Петербургское государственное

бюджсетное учреждение здравоохранения Городская клиническая больнища № 31 (Санкт-Петербург, Россия)

${ }^{3}$ Санкт-Петербургское государственное

бюджсетное учрежхдение здравоохранения Городская больница № 15 (Санкт-Петербург, Россия)

${ }^{4}$ Государственное бюджстное учреждение здравоохранения «енинградская областная клиническая больнища Министерства здравоохранения Ленинградской области» (Ленинградская область, Россия)

5 Федеральное государственное бюджсетное учрежсдение науки «Кировский научноисследовательский институт гематологии и переливания крови Федерального медикобиологического агентства России» (Киров, Россия)

${ }^{6}$ Больница Тимон (Марсель, Франция)

\section{ОСОБЕННОСТИ И РЕЗУЛЬТАТЫ} ТЕРАПИИ БОЛЬНЫХ ПЕРВИЧНОЙ МЕДИАСТИНАЛЬНОЙ В-КЛЕТОЧНОЙ КРУПНОКЛЕТОЧНОЙ ЛИМФОМОЙ

Ю.Н. Виноградова ${ }^{1}$, В.В. Рябчикова ${ }^{2}$, А.И. Чумаченко ${ }^{1}$, Е.В. Карягина ${ }^{3}$, Н.В. Медведева ${ }^{2}$, А.С. Лучинин ${ }^{5}$, Н.В. Минаева ${ }^{5}$, Т.В. Шнейдер ${ }^{4}$, М.М. Ходжибекова ${ }^{1}$, Iline Nicolas $^{6}$, H.В. Ильин ${ }^{1}$

\section{MAIN ASPECTS AND RESULT'S TREATMENT OF PRIMARY}

MEDIASTINAL B-CELL LARGE LYMPHOMA

Ю.Н. Виноградова ${ }^{1}$

Д. м.н., руководитель отдела лучевых и комбинированных методов лечения, доцент кафедры радиологии, хирургии и онкологии,

Федеральное государственное бюджетное учреждение «Российский научный центр радиологии и хирургических технологии имени академика А.М. Гранова» Министерства здравоохранения Российской Федерации. 197758, Санкт-Петербург, п. Песочный, ул. Ленинградская, д. 70. SPIN-код 8876-8936. ORCID: 0000-0002-0938-5213.

В.В. Рябчикова ${ }^{2}$ Заведующая гематологическим отделением Санкт-Петербургского государственного бюджетного учреждения здравоохранения Городская клиническая больнища № 31 . 197110, Санкт-Петербург, пр. Динамо, д. 3. SPIN-код 5879-2510. ORCID: 0000-0003-1492-5939.

\section{А.И. Чумаченко ${ }^{1}$}

Км.н., заведуюшая учебной частью, доцент кафедры радиологии, хирургии и онкологии, Российский научный центр радиологии и хирургических технологий имени академика А.М. Гранова. SPIN-код: 6179-0623.

$$
\begin{array}{r}
\text { E.в. Карягина } \\
\text { Заведуюшая гематологическим отделением Санкт-Петербургского } \\
\text { государственного бюджетного учреждения здравоохранения } \\
\text { Городская больница №15, Санкт-Петербург. } \\
\text { 198205, Санкт-Петербург, ул. Авангардная, д. } 4 . \\
\text { SPIN-код: 8158-0620. }
\end{array}
$$

Н.В. Медведева ${ }^{2}$

К.м.н., заместитель главного врача Санкт-Петербургского государственного бюджетного учреждения здравоохранения Городская клиническая больница № 31. Санкт-Петербург.

\section{А.С. Лучинин 5} Кмм.н., врач-гематолог Федерального государственного бюджетного учреждения науки Кировский научно-исследовательский институт гематологии и переливания крови Федерального медико-биологического агентства России, 610027, г. Киров, ул. Красноармейская, д. 72. SPIN-код: 6660-3985. ORCID: 0000-0002-5016-210X. 


\section{Н.В. Минаева}

К.м.н., заместитель директора, Федеральное государственное бюджетное учреждение науки Кировский научно-исследовательский институт гематологии и переливания крови Федерального медикобиологического агентства России. SPIN-кod: 8927-8003. ORCID: 0000-0002-8479-3217.

T.в. Шнейдер 4

Заведуюшая гематологическим отделением, Государственное бюджетное учреждение здравоохранения Ленинградская областная клиническая больница Министерства здравоохранения Ленинградской области, 194291, Санкт-Петербург, пр. Луначарского, 45-49.

\section{М.М. Ходжибекова ${ }^{1}$}

Длм.н, врач-радиолог, Федеральное государственное бюджетное учреждение «Российский научный центр радиологии и хирургических технологий илени академика А.М. Гранова» Министерства здравоохранения Россииской Федеращии. SPIN-код: 3999-7304. ORCID: 0000-0002-2172-5778.

\section{Н. Ильин}

Врач-интерн, департалент биостатистики и медицинских информационных технологий Больница Тимон, 13005, Франция, Марсель, ул. Сен-Пьер, 264.

\section{Н.В. Ильин ${ }^{1}$}

Д.м.н., профессор, главный научный сотрудник, профессор кафедры радиологии, хирургии и онкологии; Федеральное государственное бюджетное учреждение «Российский научный центр радиологии и хирургических технологий им. акад. А.М. Гранова» Министерства здравоохранения Российской Федеращии. SPIN-код: 2242-2112. ORCID: 0000-0002-8422-0689.

\section{J.N. Vinogradova ${ }^{1}$}

Doctor of Medicine, Head of Radiation and Combined Treatment Department, associate professor of the education department of radiology, surgery and oncology,

A.M. Granov Russian Research Center for radiology and surgical technologies; 197758, St.-Petersburg, Pesochny, Leningradskaya ul., 70. SPIN-code6 8876-8936. ORCID: 0000-0002-0938-5213

\section{V.V. Rjabchikova ${ }^{2}$}

Head of the Hematology DepartmenSt.-Petersburg State Budget Institution of Healthcare «City Clinical Hospital № 31", 197110, St.-Petersburg, pr. Dinamo, 3. SPIN-code: 5879-2510. ORCID: 0000-0003-1492-5939.

\section{A.I. Chumachenko ${ }^{1}$}

Candidate of Medicine, Chief of the educational part, associate professor of the education department of radiology, surgery and oncology; A.M. Granov Russian Research center for radiology and surgical technologies. SPIN-code: 6179-0623.

E.V. Karjagina ${ }^{3}$

Head of the Hematology Departmen St.-Petersburg State Budget Institution of Healthcare «City Clinical Hospital №15". 198205, St.-Petersburg, Avangardnaya ul., 15. SPIN-code: 8158-0620.

\section{N.V. Medvedeva ${ }^{2}$ \\ Candidate of Medicine, Deputy chiefphysician St.-Petersburg State Budget Institution of Healthcare «City Clinical Hospital № 31».}

A.S. Luchinin Scientific Institution Kirov Research Institute of Hematology and Blood Transfusion under the Federal Medical Biological Agence. 610027, Kirov, Krasnoarmeyskaya ul., 72. SPIN-code: 6660-3985. ORCID: 0000-0002-5016-210X.

N.V. Minaeva ${ }^{5}$

Candidate of Medicine, Deputyof director the Federal State-Financed Scientific Institution Kirov Research Institute of Hematology and Blood Transfusion under the Federal Medical Biological Agence, SPIN-code: 8927-8003. ORCID: 0000-0002-8479-3217.

T.V. Shnejder ${ }^{4}$

Head of the Hematology Leningrad Regional Clinical Hospital. 194291, St.-Petersburg, pr. Lunacharsky, 45-49.

M.M. Hodzhibekova ${ }^{1}$

Doctor of Medicine, radiologist A.M. Granov Russian Research center for radiology and surgical technologies. SPIN-code: 3999-7304. ORCID: 0000-0002-2172-5778. $N_{\text {. Iline }}^{6}$
Intern in medicine, service of Biostatistics and Medical
Information Technology Timone Hospital,
13005, France, Marseille, str. Saint-Pierre, 264.

N.V. Ilyin Doctor of Medicine, Professor, Chief research worker, professor of the education department of radiology, surgery and oncology; A.M. Granov Russian Research center for radiology and surgical technologies. SPIN-code: 2242-2112. ORCID: 0000-0002-8422-0689.

При первичной медиастинальной В-клеточной крупноклеточной лимфоме (ПМВКЛ) важен выбор варианта лечения с учетом результатов позитронно-эмиссионной и компьютерной томографии с 18F-ФДГ (ПЭТ/КТ) в динамике.

Цель исследования: Повышение эффективности лечения больных ПМВКЛ путем рационального использования лекарственного и лучевого компонентов терапии. 
Материал и методы: Проведен анализ результатов терапии 101 больного ПМВКЛ, получавших или только лекарственное лечение (34 больных, R-CHOP или R-DA-EPOCH) или иммунополихимиолучевую терапию (67 пациентов). На разных этапах наблюдения проводили ПЭТ/КТ.

Результаты: Полные ремиссии достигнуты у 94 (93,1\%) пациентов, в том числе при комбинированном лечении у 65 из 67 (97,0\%) больных; при только лекарственной терапии - у 29 из $34(85,3 \%)$ больных (р<0,02). Рецидивов после комбинированного лечения не было; в группе иммунополихимиотерапии (ИПХТ) у 1 больного возник рецидив в средостении ( $\mathrm{p}=0,04)$. Частота недостаточной эффективности первой линии ИПХТ была одинаковой после R-CHOP (20,0\%) или R-DA-EPOCH (30,4\%), p>0,1. После первой линии ИПХТ отсутствие метаболической активности (DC1-3) наблюдали у 48 (76,2\%) из 63 больных. Все они живы и находятся в ремиссии. У 4 из 15 (26,7\%) пациентов с ПЭТ-положительными результатами (DC4-5) после ИПХТ было неблагоприятное течение заболевания, что хуже по сравнению с таковым ПЭТ-отрицательной группы $(100 \%, \mathrm{p}<0,01)$. 5-летняя общая, беспрогрессивная и безрецидивная выживаемость больных всей группы была 91,6\%, 89,9\% и 95,5\%; при только лекарственной терапии и комбинированном лечении составила: общая - 79,6\% и 97,0\% ( $\mathrm{p}=0,005)$; беспрогрессивная - 77,5\% и 97,0\% ( $\mathrm{p}=0,005$ ); безрецидивная - 92,6\% и 100\% (p>0,1).

Заключение: Эффективность схем ИПХТ была сопоставимой. Отдаленные результаты были лучше при иммунохимиолучевом лечении; наличие ПЭТ-отрицательных данных после ИПХТ является благоприятным прогностическим признаком. Проведение лучевой терапии способствует уменьшению системной цитостатической нагрузки.

Ключевые слова: первичная медиастинальная В-клеточная крупноклеточная лимфома, лучевая терапия, ПЭТ/КТ.

The best choice of treatment of patients with primary mediastinal B-cell large lymphoma (PMBCL) is very important, taking into account the results of combined positron emission and computed tomography with 18F-FDG (PET/CT) at various stages of the disease clinical progression.

The aim: Increase the treatment success in patients with PMBCL by the rational use of the drug and radiation therapy.

Materials and methods: We analyzed therapy results of 101 patients with PMBCL who received either only immunopolychemotherapy (34 patients, R-CHOP or R-DA-EPOCH) or combined treatment with radiation (67 patients. PET/CT was performed before treatment, after immunopolychemotherapy and combination therapy.

Results: Complete remissions were achieved in 94 (93,1\%) patients, including those who received combined treatment in 65 of 67 (97,0\%) patients; and with drug therapy only - in 29 of 34 (85,3\%) patients ( $\mathrm{p}<0,02)$. Primary disease progression with subsequent unfavorable outcome was more frequent in the only drug therapy group than in the chemoradiotherapy group $(14,7 \%$ and $3,0 \%$, respectively, $p<0,02)$. There were no relapses after combined treatment, but in the drug-only group 1 patient had shown a relapse in the mediastinum $(\mathrm{p}=0,04)$ after 34 months. The frequency of low efficiency of the first line of immunopolychemotherapy was the same after R-CHOP (20,0\%) and R-DA-EPOCH (30,4\%), p>0,1. After the first line of immunopolychemotherapy, the absence of metabolic activity (DC1-3) was observed in 48 (76,2\%) of 63 examined patients. They are all alive and in remission. 4 from 15 (26,7\%) patients with PET-positive results of the study (DC4-5) after immunopolychemotherapy had an unfavorable progress of disease which is significantly worse compared to those of the PET-negative group $(100 \%, \mathrm{p}<0,01)$. 5 -year overall, progression-free and relapse-free survival of patients in the whole group was $91,6 \%, 89,9 \%$ and $95,5 \%$; with only drug therapy and combined treatment was: total $-79,6 \%$ and $97,0 \%(\mathrm{p}=0,005)$; nonprogressive $-77,5 \%$ and $97.0 \%(\mathrm{p}=0,005)$; relapse-free $-92,6 \%$ and $100 \%(\mathrm{p}>0,1)$.

Conclusion: Efficiency of first-line immunopolychemotherapy schemes was comparable. Long-term results were better with immunochaemoradiotherapy; the presence of PET-negative data after the first line of immunopolychemotherapy is a favourable prognostic sign. Radiation therapy executed in proper time helps to reduce the systemic cytostatic stress.

Keywords: primary mediastinal B-cell large lymphoma, radiotherapy, PET/CT.

\section{Введение}

$\prod$ ервичная медиастинальная В-клеточная крупноклеточная лимфома (ПМВКЛ) является экстранодальной лимфомой с поражением, прежде всего, тимуса, лимфатических узлов средостения и окружающих тканей с частым развитием синдрома верхней полой вены и редким вовлечением периферических лимфатических узлов и костного мозга. В стандартные лечебные мероприятия входят ИПХТ по программам R-CHOP, R-DA-EPOCH, R-MACOP-B и локальная лучевая терапия на оставшиеся опухолевые массы, прежде всего, в средостении. В литературе активно обсуждаются данные об эффективности в первой линии различных режимов ИПХТ, роли со-

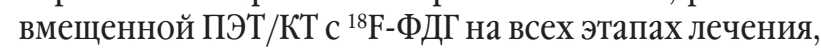

возможности ограничения лучевой терапии у ряда категорий больных $[1,2,3]$.

Известно, что в целом результаты терапии больных ПМВКЛ в значительной степени зависят от эффективности первой линии лечения - ИПХТ с или без лучевой терапии, а также при недостаточной эффективности - второй и последующих линий, включая высокодозную химиотерапию с аутологичной трансплантацией периферических гемопоэтических стволовых клеток. В последнее время разрабатываются и новые подходы в виде иммунотерапии.

Большой вклад в изучение ПМВКЛ внесли итальянские исследователи. Zinzani et al. [4] оценили результаты терапии 74 больных ПМВКЛ (2002-2011 гг.), кото- 
рым проводили R-MACOP-B + лучевую терапию только на остаточные образования, активно накапливающие

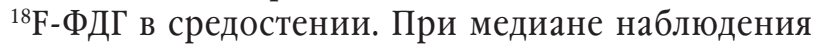
4 года полная ремиссия достигнута у $82,4 \%$ пациентов; после ИПХТ метаболически активные очаги наблюдались у 69\% больных, которые подвергались облучению. Пять больных (6,8\%) прогрессировали в пределах года от начала лечения. 10-летняя общая и беспрогрессивная выживаемость составили $82 \%$ и $88 \%$ соответственно. Не было различий в беспрогрессивной выживаемости (90\%) у больных с ПЭТотрицательными и ПЭТ-положительными данными после ИПХТ. По протоколу IELSG-26 [5] 125 больных ПМВКЛ получали ИПХТ, затем 88 из них - лучевую терапию. Полный метаболический ответ, согласно критериям Deauville (DC) соответствующий $1,2,3$ баллам после ИПХТ зафиксирован у 74\% больных, после ИПХТ + лучевая терапия - у 89\% пациентов. У 16 больных (12,8\%) с DC в 4,5 баллов после лучевой терапии отмечался неблагоприятный прогноз заболевания. При медиане наблюдения 60 мес. у всех пациентов с полным метаболическим ответом после облучения рецидивов не было. По более ранним данным этих же исследователей [6], а также американских авторов [7] при ПМВКЛ большое влияние на выживаемость оказывает метаболический объем и общий гликолитический индекс (TLG - total lesion glycolysis) опухоли.

По данным многих авторов $[5,6,8,9,10]$ первичное прогрессирование в течение первых 2-х лет от начала лечения при ПМВКЛ возникает у 6-18\% больных. Несмотря на высокую эффективность схемы R-CHOP, указывают на преимущество более интенсивной программы R-DA-EPOCH [9, 11], хотя это мнение не всеми разделяется $[12,13]$. R-DA-EPOCH, как более интенсивный режим, потенциально может быть более эффективным, но он более токсичный по сравнению c R-CHOP.

325 больных ПМВКЛ получали 6-8 циклов R-CHOP \pm лучевую терапию. При среднем периоде наблюдения 64 месяцев первичное прогрессирование наблюдали у 10,5\% пациентов, 5-летняя общая и беспрогрессивная выживаемость были 89\% и 78\% соответственно, худший прогноз был при сочетании экстранодального поражения с крупным образованием или повышенным более чем в 2 раза уровнем лактатдегидрогеназы [10]. Чешские авторы [12] наблюдали (средний период наблюдения 6,8 лет) 102 пациента ПМВКЛ (2001-2017 гг.), которые получали R-CHOP \pm лучевую терапию (назначение лучевой терапии зависело от решения лечащего врача). 5-летняя общая и беспрогрессивная выживаемость составили 86\% и 80\%; выявлено 16 рецидивов, $8 \%$ пациентов умерли. Из 71 больного с полной ремиссией, 40 получали лучевую терапию, 31 пациента только наблюдали. Оказалось, что 5-летняя беспрогрессивная выживаемость у больных с полной ремиссией и с лучевым лечением составила 97\%, а без облучения средостения $-80 \%$ (p=0,03). После лучевой терапии возникло только 2 (5\%) рецидива, а без ее применения - 6 (19,4\%) (p<0,05); общая выживаемость была одинаковой в группах с или без лучевого лечения (90-95\%).

Trneny et al. [14] показали, что из 190 больных ПМВКЛ, получавших в первой линии R-CHOP или R-DA-EPOCH, у 28\% из них необходимо было провести высокодозную химиотерапию с аутологичной трансплантацией гемопоэтических стволовых клеток. После ИПХТ у $72 \%$ больных отмечались ПЭТотрицательные результаты, 23\% - ПЭТ-положительные (остальных больных не обследовали). При медиане наблюдения 4,5 года, 5-летняя общая выживаемость составила $89,4 \%$ у больных с ПЭТ-отрицательными данными и $80 \%$ у пациентов с ПЭТ-положительным результатом $(\mathrm{p}=0,02)$. Из 136 больных с отсутствием метаболической активности опухоли 54 пациента получали лучевую терапию, а 82 больных только наблюдали; различия в 5-летней беспрогрессивной выживаемости в 10\% (95,5\% и 85,5\%) были в пользу проведения лучевой терапии с пограничными данными статистической достоверности $(\mathrm{p}=0,06)$. Тем не менее важным наблюдением было, что при отсутствии облучения средостения риск прогрессирования заболевания или смерти был повышен в 3,5 раза $(\mathrm{p}=0,05)$.

Группа исследователей British Centre Cancer из Канады [13] проанализировала результаты терапии 159 больных ПМВКЛ (медиана наблюдения 7,9 лет), из них 50 пациентов до выполнения ПЭТ/КТ получали R-CHOP и лучевую терапию, и 109 больных - R-CHOP \pm лучевую терапию в зависимости от данных промежуточного ПЭТ/КТ-исследования. У 40\% больных определялось крупное образование в средостении, у 40\% - плевральные выпоты, 37\% пациентов - имели экстранодальные поражения, $72 \%$ - повышенный уровень ЛДГ, 63\% - I-II стадии заболевания. Облучение средостения получали всего 70 (44\%) пациентов, из них 39 - в 2001-2005 гг. (без результатов ПЭТ/КТ), 31 - в более поздние годы с использованием ПЭТ/КТ-исследования. В целом у 159 больных 5-летняя общая и беспрогрессивная выживаемость составили $89 \%$ и $80 \%$ соответственно. Не было различий в отдаленных результатах групп больных, леченных в период 2000-2005 гг. и в более поздние годы. Промежуточное ПЭТ/КТ-исследование после ИПХТ выполнено у 113 больных, из них оно было отрицательным у 71 (63\%), положительным - у 42 (37\%) пациентов. Больных с клинико-метаболической ремиссией после R-CHOP только наблюдали; c остаточными ПЭТ-активными очагами - проводили лучевую терапию (у 33 из 42 пациентов, 79\%). 5-летняя общая и беспрогрессивная выживаемость 113 больных составили 94\% и 83\%; общая выживаемость пациентов с ПЭТ-отрицательными результатами без лучевой терапии и ПЭТ-положительными (с лучевой терапией) была одинаковой, однако беспрогрес- 
сивная выживаемость ПЭТ-отрицательных больных (после ИПХТ) без облучения была выше (88\%), чем пациентов с ПЭТ-положительными данными после ИПХТ, получавших лучевую терапию (74\%), p=0,03. Авторы заключают, что программа R-CHOP сама по себе очень эффективна: общая 5-летняя выживаемость 89\%. Терапия, адаптированная результатами ПЭТ/КТисследования после R-CHOP, приводит к уменьшению частоты использования лучевой терапии на $60 \%$ без ухудшения отдаленных результатов.

Опубликованы результаты самого крупного отечественного исследования - 131 больной ПМВКЛ из НМИЦ им. Н.Н. Блохина [9]. После первой линии (R-CHOP, или R-DA-EPOCH, или R-MACOP-B) у 99 пациентов проведена лучевая терапия в условиях 2D или 3D-планирования. Показано, что интенсивные режимы ИПХT - R-DA-EPOCH и R-MACOP-B - имеют преимущества перед стандартной схемой R-CHOP. При иммунохимиолучевом лечении при медиане наблюдения 37 мес. 3-летняя общая и беспрогрессивная выживаемость составили 91\% и $88 \%$ соответственно. 17 пациентов с первичным прогрессированием или ранним рецидивом в сроки до 13 мес. имели очень плохой прогноз: 12-месячная общая выживаемость не превышала 37\%. Промежуточное ПЭТ/КТ-исследование после ИПХТ имело важное прогностическое значение: 3-летняя выживаемость без прогрессирования в ПЭТ-отрицательной группе была 92\%, в ПЭТ-положительной - 26\%. В этом исследовании оценка эффекта различных режимов ИПХТ проводилась только в сочетании с лучевой терапией; отдельно непосредственную әффективность схем ИПХТ изучали только у 17 пациентов с неблагоприятным исходом заболевания: 7 пациентов - R-MACOP-B, 9 - R-CHOP, 1 больной - R-DA-EPOCH. К настоящему времени нет рандомизированных исследований по сравнительной эффективности режимов R-DA-EPOCH и R-CHOP [3].

Американские исследователи показали [15] на большой группе - 426 больных ПМВКЛ, преимущества адъювантной лучевой терапии после ИПХТ: 5-летняя общая выживаемость была 93\% при комбинированном лечении по сравнению с таковой (83\%) при только ИПХТ ( $\mathrm{p}<0,05)$. С другой стороны, 2 ретроспективных исследования [16, 17], проведенных с учетом ПЭТ/ КТ-исследования, продемонстрировали отсутствие необходимости проведения лучевой терапии после схем R-CHOP или R-DA-EPOCH.

Недавно опубликовано большое исследование из Национального онкологического института в Бетезде (США), в котором проводили лечение 93 пациентам ПМВКЛ по программе R-DA-EPOCH (1999-2016 гг.) без лучевой терапии [18]. При медиане наблюдения 8,4 года 8-летняя общая и беспрогрессивная выживаемость составили 95\% и 90,0\%; результаты были значительно лучше в ПЭТ-отрицательной группе (у 69\% пациентов), в сравнении с пациентами, у которых наблюдалась метаболическая активность опухоли в средостении после ИПХТ (ПЭТ-положительная группа - 31\% больных). При этом общая выживаемость в этих группах пациентов составили $97,7 \%$ и $84,3 \%$ соответственно ( $<0,01)$; выживаемость без прогрессирования $96 \%$ и $71 \%(\mathrm{p}<0,01)$ соответственно. Авторы утверждают, что только 5\% пациентов после программы R-DA-EPOCH нуждаются в облучении. Однако решение не использовать лучевую терапию очень ответственно и должно применяться не только на основании данных ПЭТ/КТ, но и с учетом множества других факторов.

В целом у 10-30\% первичных больных ПМВКЛ наблюдается неблагоприятный исход; у них безрецидивная выживаемость не превышает 27\% [19]. Оценка данных ПЭТ/КТ после ИПХТ осложняется тем, что могут наблюдаться ложноположительные результаты исследования, связанные с воспалительными процессами в средостении после ИПХТ [3].

В Италии (Болонья) 95 больных ПВМКЛ получали терапию за последние 20 лет по схеме (R) MACOP-B, из них лучевая терапия проведена 37 пациентам. Полная ремиссия после (R) МАСОР-В достигнута у $62 \%$, частичная ремиссия - у $27 \%$ пациентов, после лучевой терапии частичная ремиссия переходила в полную у 21 больного; в целом после химиолучевого лечения у 99,8\% пациентов достигнута ремиссия. В первые 2 года у 11 больных (11,6\%) наблюдали прогрессирование и раннее рецидивирование. 17-летняя общая выживаемость была 72\%; умерли 15 (15,8\%) пациентов [20].

Исследователи из Сингапура и Южной Кореи [21] оценили ответ применения R-CHOP или R-DA-EPOCH с или без лучевой терапии ПМВКЛ на основе ретроспективного мультицентрового анализа результатов 124 больных, получавших R-CHOP (n=41); R-CHOP + лучевая терапия $(\mathrm{n}=37)$ и R-DA-EPOCH $(\mathrm{n}=46)$. При медиане наблюдения 45 мес. 5-летняя общая и беспрогрессивная выживаемость всей группы составили 89,4\% и 82,4\% соответственно. Выживаемость без прогрессирования была выше у больных, получавших $\mathrm{R}-\mathrm{CHOP}+$ лучевая терапия или только R-DA-EPOCH по сравнению с только R-CHOP. Исключение лучевой терапии у пациентов, получавших R-CHOP, особенно у пациентов с крупным образованием в средостении, снижает результаты лечения.

Немецкие исследователи [22] доложили на заседании Американского общества клинической онкологии результаты UNFOLDER-трайла, включающего 131 больного ПМВКЛ, получивших 6 циклов R-CHOP-14 или 6 циклов R-CHOP-21, из них лучевую терапию в дозе 40 Гр проводили 82 (63\%) пациентам (43 - R-CHOP-21 и 39 - R-CHOP-14) на остаточные опухолевые очаги в средостении или экстралимфатические места поражений. 49 пациентов (R-CHOP-21 27; R-CHOP-14 - 22 больных не получали лучевое лечение. Полная ремиссия чаще (94\% против 84\%) 
была при химиолучевом лечении, также, как и 3-летняя бессобытийная выживаемость (94\% против 78\%, $\mathrm{p}=0,007)$, различий в общей и беспрогрессивной выживаемости не было в группах только лекарственного или комбинированного лечения. Различий в непосредственной эффективности схем R-CHOP-21 и R-CHOP-14 также не получено. В целом по группе 3-летняя общая выживаемость больных, получавших R-СНОР, была высокой - 96\%.

Таким образом, представляется актуальным изучение непосредственной эффективности наиболее используемых программ ИПХТ - R-CHOP и R-DA-EPOCH; роль и необходимость лучевой терапии при этих

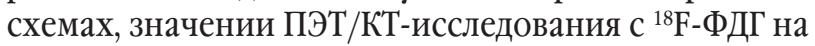
всех этапах течения заболевания; роль лучевой терапии у больных с ПЭТ-отрицательными данными после ИПХТ; необходимость второй линии лекарственного лечения у больных, начавших терапию с R-CHOP или R-DA-EPOCH.

\section{Цель исследования}

Повышение эффективности лечения больных ПмВКЛ путем рационального использования лекарственного и лучевого компонентов терапии.

\section{Материал и методы}

В исследование был включен 101 больной ПМВКЛ в возрасте 18-64 лет (средний возраст - 37,5 лет), из них мужчин - 42, женщин - 59. Все пациенты были с впервые поставленным диагнозом, проходили лечение в РНЦРХТ и других лечебных учреждениях Северо-Запада и включались в исследование только после проведения первой линии терапии независимо от ее результата. У всех пациентов диагноз был поставлен с помощью иммуноморфологического исследования опухолевой ткани. Стадирование больных выполняли согласно классификации, принятой в Анн-Арборе (США) в 1971 г. [23] с учетом рекомендаций, выработанных в Коствольде (Великобритания) и опубликованных в 1989 г. [24]. Медиана наблюдения всей группы больных ПМВКЛ составила 48,0 \pm 3,6 мес., т.е. в среднем 4 года. В 61 (60,4\%) наблюдении был локальный опухолевый процесс в пределах средостения и, в ряде случаев, с прорастанием органов грудной клетки (I стадия - 8, IIE стадия - 53 пациента), у 5 (5,0\%) больных установлена III стадия с поражением лимфатических узлов ниже диафрагмы или селезенки, IV стадия у 35 (34,6\%) пациентов или со значительным местным распространением на легкое (легкие) - 17 больных, перикард, плевру, переднюю грудную стенку с прорастанием грудины (13 больных) или поражением костей (6 больных), печени (6 больных); костного мозга - 2 больных. Крупный очаг (> 10 см) в средостении наблюдали у 50 (49,5\%) пациентов; синдром сдавления верхней полой вены был у 44 (43,6\%) больных, признаки специфической интоксикации определяли у 49 (48,5\%) пациентов.

Согласно критериям МПИ - возраст старше 60 лет, повышение уровня ЛДГ, ECOG 2-4 степени, III-IV стадия заболевания, наличие более 1 экстранодального очага поражения - выделены 4 группы риска раннего прогрессирования: низкая (0-1 фактор), промежуточная низкая - 2 фактора, промежуточная высокая - 3 фактора, высокая - 4-5 факторов. 20 $(19,8 \%)$ пациентов входили в группу низкого риска, 52 (51,5\%) - промежуточного, 29 (28,7\%) - высокого риска прогрессирования.

На разных этапах наблюдения (до лечения, через 2-3 недели после лекарственной терапии, через 3 мес. после лучевого лечения) проводили ПЭТ/КТ-

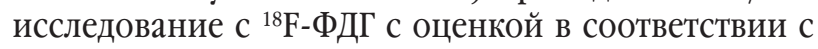
критериями Международной рабочей группы [25]. Больные согласно принятой практики клиник и историческому этапу получали один из двух режимов ИПХТ: R-CHOP - 45 пациентов или R-DA-EPOCH - 56 больных. Эти группы были сопоставимы по частоте факторов риска прогрессирования (табл. 1).

Через 3-4 недели после завершения 4-6 курсов ИПХТ 67 (66,3\%) больным в плане первичного лечения проводили лучевую терапию, в основном, на базе РНЦРХТ на линейных ускорителях электронов (Phillips SL-75-5; Elekta Precise, Elekta Axesse) тормозным излучением фотонов с энергией 6 МэВ обычным фракционированием с применением $2 \mathrm{D}$-конвенциальной (18 пациентов) и с 2013 г. 3D-конформной лучевой терапии (49 больных). Облучению подвергали область средостения в суммарной очаговой дозе 30-36 Гр в зависимости от наличия остаточной опухоли, определяемой по данным КТ или ПЭТ/КТ. При 2D-лучевой терапии в поле облучения,

Распределение групп риска прогрессирования больных при разных схемах иммунополихимиотерапии

\begin{tabular}{|l|c|c|c|c|}
\hline \multicolumn{1}{|c|}{ Группы риска } & R-CHOP & R-DA-EPOCH & Bсего & p \\
\hline Низкий & $10(22,2 \%)$ & $10(17,8 \%)$ & $20(19,8 \%)$ & $>0,1$ \\
\hline $\begin{array}{l}\text { Промежуточный } \\
\text { суммарно) }\end{array}$ & $22(48,9 \%)$ & $30(53,6 \%)$ & $52(51,5 \%)$ & $>0,1$ \\
\hline Высокий & $13(28,9 \%)$ & $16(28,6 \%)$ & $29(28,7 \%)$ & $>0,1$ \\
\hline Всего & $45(100 \%)$ & $56(100 \%)$ & $101(100 \%)$ & \\
\hline
\end{tabular}


кроме средостения, включали лимфатические узлы ворот легких, ранее пораженные отделы легочной ткани. В ряде случаев облучению подвергались остаточные опухолевые массы в других регионах.

С 2014 г. при 3D-конформной лучевой терапии объем облучения средостения устанавливался согласно рекомендациям International Lymphoma Radiation Oncology GROUP (ILROG) по границам involved site radiotherapy (IRST) - облучение пораженных мест, как правило, уменьшающим объемы облучения по сравнению с $2 \mathrm{D}$-конвенциальной лучевой терапией за счет более точного определения с помощью КТ или ПЭТ/КТ GTV (Gross tumor volume), CTV (Clinical target volume) и PTV (Planning target volume), т.е. основного объема опухоли, клинического и планируемого объемов мишени [26].

Распределение больных, получавших или не получавших лучевую терапию при разной первичной программе ИПХТ, представлено в таблице 2.

Из нее видно, что как при схеме R-CHOP, так и при R-DA-EPOCH количество больных, получавших лучевую терапию, достоверно не различалось.

Медиана наблюдения группы иммунохимиолучевого лечения составила 49,5 $\pm 2,4$ мес., а только ИПХТ - 44,6 $\pm 6,5$ мес. (p>0,1). Оценку непосредственных результатов терапии осуществляли поэтапно, как только после ИПХТ, так и после комбинированного лечения согласно критериям Cheson [27, 28, 29]. При статистической обработке материала использовали критерии t Стьюдента, точный метод Фишера; оценку актуриальной общей, беспрогрессивной и безрецидивной выживаемости проводили моментным методом с применением теста Mantel-Haenszel (критерий Logrank).

\section{Результаты}

В целом полные ремиссии достигнуты у 94 (93,1\%) из 101 больного, в том числе при комбинированном лечении у 65 из 67 (97,0\%) пациентов; при только лекарственной терапии - у 29 (85,3\%) из 34 больных $(\mathrm{p}<0,02)$. Неблагоприятное течение заболевания с последующей смертью, в основном, в течение первых двух лет наблюдения было всего у 7 из 101 (6,9\%) больного, в том числе у 2 из 67 пациентов $(3,0 \%)$ паци- ентов при химиолучевом лечении (прогрессирование заболевания в течение 16 и 18 мес.) и у 5 из 34 (14,7\%) больных в группе лекарственной терапии $(\mathrm{p}<0,02)$ в результате первичного прогрессирования заболевания в течение $10,15,18,19$ и 30 месяцев. Кроме того, в группе только ИПХТ 1 больной (К) умер в ремиссии через 67 месяцев наблюдения от миелодиспластического синдрома; у 1 пациента этой же группы из 29 больных, вошедших в ремиссию (3,5\%), через 34 мес. возник поздний рецидив в средостении, он получает терапию и наблюдается в течение 80 месяцев. Рецидивов в группе комбинированного лечения не было ( $\mathrm{p}=0,04)$. Таким образом, в целом в группе лекарственной терапии погибло 6 из 34 (17,7\%) пациентов, этот показатель больше такового группы химиолучевой терапии $(3,0 \%, \mathrm{p}<0,01)$. Погибшие в результате прогрессирования заболевания 7 больных были из группы высокого (4) и промежуточного (3 пациента) риска.

Нами проанализирована частота недостаточной эффективности различных программ первой линии терапии, после которой наблюдалась или частичная ремиссия, или стабилизация заболевания или его прогрессирование; в результате этого требовалась вторая и, в ряде случаев, последующие линии лечения, включая аутологичную трансплантацию гемопоэтических клеток.

Во всей группе было 26 таких пациентов $(25,7 \%)$, что представлено в таблице 3.

Из нее следует, что из 9 пациентов с неблагоприятным течением заболевания 4 больных начинали лечение с программы R-CHOP, 5 - c R-DA-EPOCH. После 1 линии они получали вторую линию (DHAP, ICE), 3 пациента из них в дальнейшем - высокодозную химиотерапию с аутологичной трансплантацией гемопоэтических стволовых клеток, 2 больных - лучевое лечение в плане терапии спасения. Таким образом, у больных с неблагоприятным течением заболевания неэффективность в целом первой и последующих линий лекарственной терапии наблюдали у 4 из 45 $(8,9 \%)$ больных после программы R-CHOP и у 5 из 56 (8,9\%) пациентов после программы R-DA-EPOCH ( $>0,1)$, т.е. была одинаковой. Далее, у 17 впоследствии успешно леченных больных при недостаточной

Таблища 2.

\section{Распределение больных, получавших или не получавших лучевую терапию} при разных схемах иммунополихимиотерапии

\begin{tabular}{|l|c|c|c|}
\hline \multicolumn{1}{|c|}{ Схемы ИПХТ } & ЛТ+ & ЛТ- & Всего \\
\hline R-СНОР & $34(50,8 \%)^{*}$ & $11(32,4 \%)^{*}$ & $45(44,5 \%)$ \\
\hline R-DA-ЕРОСН & $33(49,2 \%)^{* *}$ & $23(67,6 \%)^{* *}$ & $56(55,5 \%)$ \\
\hline Всего & $67(100 \%)$ & $34(100 \%)$ & $101(100 \%)$ \\
\hline
\end{tabular}

*различия не достоверны $(\mathrm{p}>0,1)$

** различия не достоверны 
Таблица 3.

Распределение больных с различныли исходали заболевания, потребовавиих вторую и последующие линии лекарственной терапии

\begin{tabular}{|l|c|c|c|}
\hline \multirow{2}{*}{$\begin{array}{c}\text { Программа } \\
\text { первой линии терапии }\end{array}$} & \multicolumn{2}{|c|}{ Исход заболевания } & \multirow{2}{*}{ Всего } \\
\cline { 2 - 3 } больных \\
\hline R-СНОР & Неблагоприятный & 2 & 5 \\
\hline R-СНОР+ЛТ & 3 & 3 & 4 \\
\hline R-DA-ЕРОСН & 1 & 7 & 11 \\
\hline R-DA-ЕРОСН+ЛT & 4 & 5 & 6 \\
\hline Всего больных & 1 & 17 & 26 \\
\hline
\end{tabular}

эффективности первой линии (R-CHOP или R-DAEPOCH) дальнейшая терапия была все же эффективной. Так, в группе комбинированного лечения у 3 пациентов после неэффективной R-CHOP и у 5 - после неэффективной R-DA-EPOCH применяли 2 линию и затем у четырех использовали высокодозную химиотерапию с аутологичной трансплантацией гемопоэтических стволовых клеток, после чего всем пациентам проводили лучевую терапию с последующей длительной полной ремиссией в течение 26,32 , 43, 43, 71, 71, 80 и 118 месяцев. В группе только ИПХТ без лучевой терапии у пациентов с благоприятным исходом заболевания вторая линия терапии потребовалась 9 пациентам (у 5 из них - и высокодозная химиотерапия с аутологичной трансплантацией гемопоэтических стволовых клеток), в том числе, у 2 из $45(4,4 \%)$ - с ранее проведенной программой R-CHOP, и у 7 из 56 (12,5\%) больных - после R-DA$\mathrm{EPOCH,} \mathrm{p}=0,06$. У всех достигнута полная ремиссия в течение $18,20,24,24,51,71,78,95,125$ месяцев. Таким образом, при благоприятном исходе заболевания в целом по группе больных ПМВКЛ дополнительные линии лекарственного лечения были необходимы у 5 из 45 (11,1\%) больных с программой R-CHOР в первой линии и у 12 из $56(21,4 \%)$ пациентов с программой R-DA-EPOCH ( $\mathrm{p}=0,08)$. Всего - с учетом больных с благоприятным и неблагоприятным течением заболевания - необходимость дополнительных линий лекарственного лечения была у 9 из 45 (20,0\%) больных после программы R-CHOP и у 17 из $56(30,4 \%)$ пациентов - после R-DA-EPOCH (p>0,1), т.е. в целом по группе с учетом применения лучевой терапии непосредственная эффективность программ первичной ИПХT (R-CHOP или R-DA-EPOCH) не различалась. Мы оценили отдельно результаты терапии группы из 34 пациентов, получавшей только лекарственное лечение (без лучевой терапии): 26 больных со схемой R-DA-EPOCH и 8 пациентов с программой R-CHOP. Из 8 пациентов, начавших лечение с программы R-CHOP, 5 больным потребовалась 2 линия терапии (62,5\%); из 26 пациентов, начавших лечение с программы R-DA-EPOCH 2 линия терапии была необходима 9 больным (34,6\%), $\mathrm{p}=0,08$, т.е. частота применения дополнительного лекарственного лечения также не различалась. 5-летняя общая выживаемость этих подгрупп также были одинаковыми 81,0\% и 75,5\% ( $>>0,1)$ соответственно. В целом необходимость дополнительной системной цитостатической нагрузки в виде дополнительных линий лекарственного лечения была значительно (более, чем в 3 раза) ниже при комбинированном лечении (у 10 из 67 больных, $14,9 \%)$, чем при только лекарственной терапии (у 16 из 34 пациентов, 47,5\%), p<0,05. В группе комбинированного лечения 5-летняя общая и беспрогрессивная выживаемость с различными схемами ИПХТ (R-CHOP + лучевая терапия и R-DA-EPOCH \pm лучевая терапия) были одинаковыми - 97,1\% и 96,9\%; p<0,1.

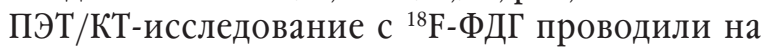
разных этапах заболевания: до лечения (22 больным), после лекарственного лечения (63 пациентам), после иммунохимиолучевой терапии (44 больным). До лечения наличие выраженной метаболической активности заболевания в средостении по данным показателя стандартизированного уровня захвата $\left(\mathrm{SUV}_{\max }\right)$ с интенсивностью накопления от 11 до 24 было у всех обследованных в этот период пациентов. После ИПХТ отсутствие метаболической активности согласно критериям Deauville $(1,2,3$ баллов) наблюдали у 48 (76,2\%) из 63 обследованных больных; по 24 пациента, получавших R-CHOP или R-DA EPOCH. Bce они живы и находятся в ремиссии, но 5 пациентам из них потребовалась после первой линии ИПХТ вторая линия лекарственной терапии, и затем, 2 пациентам была для консолидации ремиссии впоследствии проведена лучевая терапия. 43 из 48 больных (89,6\%) вторая линия терапии не потребовалась, но из них 36 пациентам проводили в плане первичного лечения лучевую терапию на остаточные образования в средостении для консолидации ремиссии. Таким образом, в ПЭТ-отрицательной группе облучение получили 38 из 48 (79,2\%) больных. Следует отметить, что из 10 ПЭТ-отрицательных после ИПХТ больных, не получавших лучевую терапию, 4 из них (40,0\%) потребовалась вторая линия лекарственного лечения для достижения ремиссии; а в ПЭТ-отрицательной группе 38 больных, которым проводили облучение, вторая линия лекарственной терапии была только у $4(10,5 \%, p=0,02)$ пациентов, т.е. своевременное проведение лучевого лечения способствует уменьшению системного цитостатического компонента терапии. 
У 4 из 15 больных с ПЭТ-положительными результатами исследования (DC 4, 5 баллов) после иПХТ (26,7\%), несмотря на проводимую в дальнейшем второю линию терапии, было прогрессирование заболевания и смерть на втором году наблюдения. У остальных 11 пациентов с ПЭТ-положительными данными после ИПХТ прогноз был благоприятным, но 5 пациентам потребовалась вторая линия терапии с последующей аутологичной трансплантацией гемопоэтических стволовых клеток (у 2 пациентов из них) и дополнительно лучевой терапией (у 2 больных).

Из 15 ПЭТ-положительных после ИПХТ пациентов у 9 было необходимо дальнейшее интенсивное лечение, в том числе у 4 больных с неблагоприятным исходом заболевания, но 6 пациентам было достаточно для благоприятного исхода первой линии терапии, включающей ИПХТ и лучевое лечение. Из 15 больных ПЭТ-положительной группы после ИПХТ 5 не получали лучевую терапию (у 2 из них было прогрессирование заболевания и смерть через 10 и 18 мес.), у 10 пациентов лучевое лечение входило в программу терапии; из них 2 больных погибли при прогрессировании болезни через 16 и 18 мес. Таким образом, В ПЭТ-положительной группе у 4 пациентов из 15 (26,7\%) было неблагоприятное течение заболевания; эти результаты достоверно хуже по сравнению с таковыми ПЭТ-отрицательной группы (100\%, p<0,01), что свидетельствует о значительном прогностическом значении промежуточного ПЭТ/КТ-исследования при положительном его результате (DC 4, 5). Следует указать, что в ПЭТ-отрицательной группе результаты были одинаково высокими (общая и беспрогрессивная выживаемость - 100\%) независимо от применения лучевого лечения при одинаковом среднем времени наблюдения больных ПЭТ-отрицательной группы с наличием или отсутствием лучевой терапии $(28,0 \pm 8,5$ мес. и $21,7 \pm 5,2$ мес., $\mathrm{p}>0,1)$.

После завершения лучевой терапии полный метаболический ответ зафиксирован у 42 из 44 (95,5\%) обследованных больных, все они находятся в длительной ремиссии. Выраженная метаболическая активность опухоли в средостении после его облучения (5 баллов) была у 2 пациентов, что сопутствовало плохому прогнозу: прогрессирование заболевания и смерть больных через 16 и 18 месяцев. У всех пациентов с частичной ремиссией после ИПХТ и завершения лучевой терапии получен полный ответ, который являлся благоприятным прогностическим признаком.

5-летняя общая, беспрогрессивная и безрецидивная выживаемость всей группы 101 больного составили 91,6\%, 89,9\% и 95,5\% соответственно. 5-летняя общая выживаемость групп только ИПХТ или комбинированного лечения составила 79,6\% и 97,0\% соответственно ( $\mathrm{p}=0,005), 5$-летняя беспрогрессивная выживаемость этих же групп 77,5\% и 97,0\%, p=0,005, безрецидивная - 92,6\% и 100\% (p>0,1). 5-летняя общая выживаемость ПЭТ-отрицательных и ПЭТ- положительных после ИПХТ пациентов составили $100 \%$ и $72,1 \%$ соответственно ( $\mathrm{p}=0,004)$, те же данные получены и по беспрогрессивной выживаемости.

\section{Обсуждение}

Как следует из литературных источников, количество больных после первой линии ИПХТ (R-CHOP, R-DA-EPOCH, (R)-MACOP-B) с отсутствием метаболической активности заболевания в средостении по данным ПЭТ/КТ-исследования колеблется в пределах 69-84\%, причем в доступных нам исследованиях одна и та же схема давала различный эффект. В то же время авторы не оценивали непосредственную әффективность каждой схемы ИПХТ в одном исследовании с учетом частоты метаболической ремиссии и необходимости дополнительной лекарственной терапии, включая высокодозную химиотерапию с аутологичной трансплантацией гемопоэтических стволовых клеток.

Важная особенность нашего исследования - это включение пациентов только после проведения первой линии терапии независимо от ее результатов. Она состояла только из циклов или R-CHOP или R-DA-EPOCH (группа лекарственного лечения) или сочетание ИПХТ и лучевой терапии независимо от результатов промежуточного ПЭТ/КТ-исследования (группа комбинированной терапии). Известно, что ряд больных с первичным прогрессированием на фоне незавершенного первого этапа лекарственного лечения часто не являются кандидатами для лучевого лечения и не получают его в плане первичной терапии. Указанный нами критерий включения важен для сопоставимости изучаемых групп.

Нами впервые получены в одном исследовании сравнительные данные об непосредственной эффективности схем R-CHOP и R-DA-EPOCH с учетом как необходимости дополнительных линий лекарственной терапии в каждой из этих групп, так и различных исходов заболевания. Показано, что непосредственная эффективность R-CHOP и R-DAEPOCH одинаковы, что отражается и в равных показателях общей и беспрогрессивной выживаемости, как в группе только ИПХТ, так и комбинированного лечения. В нашей группе больных промежуточное ПЭТ/КТ-исследование было отрицательным у 76,2\% больных, общая и беспрогрессивная выживаемость этой группы составили 100\%. Результаты терапии пациентов с ПЭТ-положительными после ИПХТ данными достоверно хуже таковых у пациентов ПЭТ-отрицательной группы. С учетом максимальной сопоставимости групп по критерию включения больных после завершенной первой линии терапии нами показано преимущество комбинированного лечения перед только лекарственной терапией в плане лучших отдаленных результатов и меньшего числа больных, подвергающихся значительной системной цитостатической нагрузке. 


\section{Заключение}

В целом результаты ИПХТ больных ПМВКЛ превышают таковые пациентов при только лекарственном лечении. Непосредственная эффективность программ R-CHOP и R-DA-EPOCH сопоставима; после их проведения частота использования дополнительных линий терапии, необходимых для достижения полной ремиссии, одинакова. Отдаленные результаты лечения по критериям общей и беспрогрессивной выживаемости пациентов, получавших только R-CHOP или R-DAEPOCH, одинаковы; также показатели выживаемости не различались в группах R-CHOP с лучевой терапией и R-DA-EPOCH с включением лучевого лечения. Эти данные показывают отсутствие принципиальных различий в результатах лечения больных, начавших терапию с R-CHOP или R-DA-EPOCH, но важно использование последующей лучевой терапии для повышения эффективности. Результаты ПЭТ/КТ с

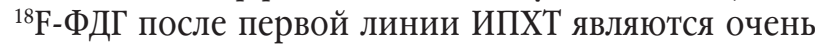
значимыми для прогноза дальнейшего течения заболевания. Возможность исключения лучевой терапии у ПЭТ-отрицательных после ИПХТ больных является очень ответственным решением, требует большого рандомизированного исследования и пока не может быть рекомендовано в клиническую практику.

\section{Список литературы}

1.Kocurek A., Malkowski B., Giza A., Jurcza K.W. Primary mediastinal B-cell lymphoma - metabolic and anatomical features in ${ }^{18} \mathrm{FDG}$ - PET/CT and response to therapy // Contemp Oncol (Pozn). - 2016. - Vol. 20, № 4. - P. 297-301.

2. Roth L.G. How I treat primary mediastinal B-cell lymphoma // Blood. - 2018. Aug 23. - Vol. 132, № 8. - P. 782-790.

3. Lees Ch., Keane C., Gandri M.K., Gunawardana J. Biology and therapy of primary mediastinal B-cell lymphoma: current status and future directions // Br J Haematol. - 2019 Apr. - Vol. 185, № 1. - P. 25-41.

4. Zinzani P., Casadei B., Pellegrini C., Broccoli A., Derenzini E., Gandolfi L., Quirini F., Stefani G., Narducci R., Tschon M., Argnani L., Stefoni $V$. The role of rituximab and PET in the treatment of primary mediastinal large B-cell lymphoma. // Hematol Oncol. - 2013. - Vol. 31, suppl. 1. - P. 128-129.

5. Ceriani L., Martelli M., Zinzani P., Ferreri A., Vitolo U., Stelitano C., Gotti M., Cabras M., Rigacci L., Rusconi C., Merli F., Pinotti G., Mannina D., LuminariS., Stathis A., Giovanella L., Zucca E., Johnson P.W. Use of the Lugano classification criteria for PET/CT assessment of primary mediastinal B-cell lymphoma after immunochemotherapy and irradiation in the IELSG-26 study // Hematol Oncol. - 2015. - Vol. 33, suppl. 1. - P. 143.

6. Ceriani L., Zucca E., Zinzani P., Ferreri A., Vitolo U., Stelitano C., Brusamolino E., Cabras G., Rigacci L., Salvi F., Gargantini L., Balzarotti M., Merli F., Pinotti G., Montoto S., Lopez-GuillermoA., Cavalli M., Giovanella L., Johnson P.W., Martelli M. Prognostic value of ${ }^{18} \mathrm{FDG}$ baseline functional PET parameters in primary diffuse large B-cell lymphoma // Hematological Oncology. - 2013. - Vol. 31, suppl. 1. - P. 187-188.

7. Pinnix C.C., Ng A.K., Dabaja B.S., Milgrom S.A., Gunther J.R., Fuller C.D., Smith G.L., Yebia Z.A., Qiao W., Wogan C.F., Akbtari M., Mawlawi O., Medeiros L.J., Chuang H.H., Martin-Doyle W., Armand P., LaCasce A.S., Oki Y., Fanale M., Westin J., Neelapu S., Nastoupil L. Position emission tomography-computed tomography predictors of progression after DA-R-EPOCH for PMBCL // Blood Adv. - 2018. - Vol. 2, № 11. P. 1334-1343.

8. Tavares M., Simas A.M., Santo A.E., Domingues N., Oliveira I., Moreira I., Martins A., Viterbo L., Henrique R., Chacim S., MarizJ. Favorable outcome of primary mediastinal diffuse large B-cell lymphoma: a Portugal Single-Center Experience // Hematol Oncol. - 2015. - Vol. 33, suppl. 1. - P. 294-295.

9. Трофимова О.П., Заводнова И.З., Тумян Г.С., Прямикова Ю.И., Волкова Н.В., Зайченко О.С., Назаренко А.В. Современная лучевая терапия в мультимодальном лечении больных первичной медиастинальной (тимической) В-крупноклеточной лимфомой (результаты лечения 131 больного в ФГБУ «НМИЦ онкологии им. Н.Н. Блохина» Минздрава России) // Современная онкология. - 2018. - Т. 20, № 4. - С. 5-15.

10. Vassilakopoulos T.P., Papageorgiou S.G., Michail M., Angelopoulou M.K., Kourti G., Kalpadakis C., Kotsopoulou M., Leonidopoulou T., Konstantinidou P., Kotsianidis I., Boutsis D., Michali E., Sachanas S., Terpos E., Karinakis G., Poziopoulos C., Vadikolia C., Pigaditou A., Vrakidou E., Anargyrou K., Symeonidis A., Stefanoudaki E., Hadjibarissi E., Papaioannou M., Gainaru G., Tsirogianni M., Katodritou E., Karmiris T., Variami E., Pappa V., Dimopoulos M., Roussou P., Panaytidis P., Konstantopoulos K., Pangalis G.A. Prognostic factors (pfs) in primary mediastinal large b-cell lymphoma (pmlbcl) treated with rituximab-chop (rchop) \pm radiotherapy (rt) // Hematological Oncology. - 2017. - Vol. 37, Issue S2. P. 266-267.

11.Dunleavy K. Primary mediastinal B-cell lymphoma: biology and evolving therapeutic strategies // Hematology Am Soc Hematol Educ Program. - 2017, Dec 8; - 2017. Vol. 1. - P. 298-303.

12. Salek D., Janikova A., Michalka J., Miculkova E., Hadrabova M., Folber F., Kral Z., Mayer J. First-line adjuvant radiotherapy improves PFS in primary mediastinal B-cell lymphoma - a retrospective analysis of 102 patients from a single center // Hematological Oncology. - 2019. - Vol. 37, suppl. 2. - P. 458.

13. Hayden A., Tonseth P., Villa D., Gerrie A.S., Scott D.W., Freeman C.L., Slack G.W., Parsons C., Pickles T., Wilson D., Connors J.M., Sebn L.H., Savage K.L. Outcome of primary mediastinal large B-cell lymphoma in the rituximab era: impact of a PET-guided approach // Hematological Oncology. - 2019. - Vol. 37, suppl. 2. - P. 156-157. 
14. Trneny M., Polgarova K., Janikova A., Belada D., Prochazka V., Duras J., Mocikova H., Steinerova K., Campr V., Blabovcova P., Petrova M., Zogala D., Ptachik V. Is it radiotherapy necessary for primary mediastinal B-cell lymphoma (PMBL) patients achieving PET negativity after immunochemotherapy? // Hematological Oncology. - 2019. Vol. 37, suppl. 2. - P. 267-268.

15.Jackson M.W., Rusthoven C.G., Jones B.L., Kamdar M., Rabinovitch R. Improved survival with combined modality therapy in the modern era for primary mediastinal B-cell lymphoma // Am J Hematol. - 2016. - Vol. 91, № 5. - P. 476-80.

16. Savage K.J., Al-Rajbi N., Voss N., Paltiel C., Klasa R., Gascoyne R.D., Connors J.M. Favorable outcome of primary mediastinal large B-cell lymphoma in a single institution: the British Columbia experience // Ann Oncol. - 2006. Vol. 17, № 1. P. 123-30.

17. Dunleavy K., Pittaluga S., Maeda L.S., Advani R., Chen C.C., Hessler J., Steinberg S.M., Grant C., Wright G., Varma G., Staudt L.M., Jaffe E.S., Wilson W.H. Dose-adjusted EPOCH-rituximab therapy in primary mediastinal B-cell lymphoma // N Engl J Med. - 2013. - Vol. 368, № 15. - P. 1408-16.

18. Milani Ch., Advani R., Roschewski M., Walters K.M., Chen C.C., Baratto L., Ablman M.A., Miljkovic M.D., Steinberg S.M., Lam J., Shovlin M., Dunleavy K., Pittaluga S., Jaffe E.S., Wilson W.H. End of treatment and serial PET imaging in primary mediastinal B-cell lymphoma following dose-adjusted EPOCH-R: a paradigm shift in clinical decision making // Haematologica. - 2018. - Vol. 103, № 8. - P. 1337-1344.

19. Aoki T., Shimada K., Suzuki R., Izutsu K., Tomita A., Maeda Y., TakizawaJ., Mitani K., Igarashi T., Sakai K., Miyazaki K., Mihara K., Obmachi K., Nakamura N., Takasaki H., Kiyoi H., Nakamura S., Kinoshita T., Ogura M. High-dose chemotherapy followed by autologous stem cell transplantation for relapsed/refractory primary mediastinal large B-cell lymphoma // Blood Cancer J. - 2015. - Vol. 5, № 12. - P. e372.

20. Broccoli A., Casadei B., Stefoni V., Pellegrini C., Quirini F., Tonialini L., Morigi A., Marangon M., Argnani L., Zinzani P.L. The treatment of primary mediastinal large B-cell lymphoma: a two decades monocentric experience with 98 patients // BMC Cancer. - 2017. - Vol. 17, № 1. - P. 276.

21. Chan E.L., Kob L.P., Lee J., De Mel S., Jeyasekharan A., Liu X., Tang T., Lim S.T., Tao M., Quek R., M.F.B.H. Ras, Lee Y.S., Diong C., Tan D., Kim S.J., Chee Y.L., Poon L.M. Real world experience of R-CHOP with or without consolidative radiotherapy vs DA-EPOCH-R in the first-line treatment of primary mediastinal B-cell lymphoma // Cancer Med. 2019. - Vol. 8, № 10. - P. 4626-4632.

22. Held G., Thurner L., Poeschel V., Berdel Ch., Ott G., Schmidt Ch., Viardot A., Borchmann P., Shpilberg O., Nickelsen M., Federico M., Brown P., Murawski N., Trumper L.H., Schmidberger H., Ruebe Ch., Fleckenstein J., Schmitz N., Loeffler M., Ziepert M. Role of radiotherapy and dose-densification of R-CHOP in primary mediastinal B-cell lymphoma: a subgroup analysis of the unfolder trial of the German Lymphoma Alliance (GLA) // J Clin Oncol. - 2020. - Vol. 38, № 15 suppl. - P. 8041-8041.

23. Carbone P.P., Kaplan H.S., MusshoffK., Smithers D.W., Tubiana M. Report of the Committee on Hodgkin's disease staging classification // Cancer Res. - 1971. - Vol. 31, № 11. - P. 1860-1.

24.Lister T.A., CrowtherD., Sutcliffe S.B., Glatstein E., Canellos G.P., Young R.C., Rosenberg S.A., Coltman C.A., Tubiana M. Report of a committee convened to discuss the evaluation and staging of patients with Hodgkin's disease: Cotswolds Meeting // J Clin Oncol. - 1989. - Vol. 7, № 11. - P. 1630-6.

25. Cheson B.D., Fisher R.I., Barrington S.F. Recommendations for initial evaluation, staging, and response assessment of Hodgkin and non-Hodgkin lymphoma: the Lugano classification J Clin Oncol. - 2014. - Vol. 32, № 27. - P. 3059-68.

26.Specht L., Yahalom J., Illidje T., Berthelsen A.K., Constine L.S., Eich H.T., Girinsky Th., Hoppe R.T., Mauch P., Mikhaeel N.G., $\mathrm{Ng}$ A. ILROG. Modern radiation therapy for Hodgkin lymphoma: field and dose guidelines from the international lymphoma radiation oncology group (ILROG) // Int J Radiat Oncol Biol Phys. - 2014. - Vol. 89, № 4. - P. 854-62.

27. Cheson B.D., Horning S., Coiffer B., Shipp M., Fisher R., Connors J. Report of an international workshop to standardize response criteria for non-Hodgkin's lymphomas. NCI Sponsored International Working Group. J Clin Oncol. - 1999. - Vol. 17, № 4. - P. 1244.

28. Cheson B.D., Pfistner B., Juweid M., Gascoyne R.D., Specht L., Horning S.J. Revised response criteria for malignant lymphoma. J Clin Oncol. - 2007. - Vol. 25, № 5. - P. 579-86.

29. Cheson B.D. PET/CT in lymphoma: current overview and future direction // Semin Nucl Med. - 2018. Vol. 48, № 1. P. 76-81.

\section{References}

1.KocurekA., Malkowski B., GizaA., Jurcza K.W. Primary mediastinal B-cell lymphoma - metabolic and anatomical features in ${ }^{18} \mathrm{FDG}$ - PET/CT and response to therapy. Contemp Oncol (Pozn). 2016; 20(4): 297-301. doi: 10.5114/ wo.2016.61849. PMCID: PMC5032157. PMID: 27688726.

2. Roth L.G. How I treat primary mediastinal B-cell lymphoma. Blood. 2018 Aug 23; 132(8): 782-790. doi: 10.1182/ blood-2018-04-791566. PMCID: PMC6634954. PMID: 29976557.

3. Lees Ch., Keane C., Gandri M.K., Gunawardana J. Biology and therapy of primary mediastinal B-cell lymphoma: current status and future directions. Br J Haematol. 2019 Apr; 185(1): 25-41. doi: 10.1111/bjh.15778. PMCID: PMC6594147. PMID: 30740662.

4. Zinzani P., Casadei B., Pellegrini C., Broccoli A., Derenzini E., Gandolfi L., Quirini F., Stefani G., Narducci R., Tschon M., Argnani L., Stefoni $V$. The role of rituximab and PET in the treatment of primary mediastinal large B-cell lymphoma. Hematol Oncol 2013; 31, suppl. 1: 128-129. doi: 10.1002/hon. 
5. Ceriani L., Martelli M., Zinzani P., Ferreri A., Vitolo U., Stelitano C., Gotti M., Cabras M., Rigacci L., Rusconi C., Merli F., Pinotti G., Mannina D., LuminariS., Stathis A., Giovanella L., Zucca E., Johnson P.W. Use of the Lugano classification criteria for PET/CT assessment of primary mediastinal B-cell lymphoma after immunochemotherapy and irradiation in the IELSG-26 study. Hematol Oncol 2015; 33, suppl. 1: 143. doi: 10.1002/hon.

6. Ceriani L., Zucca E., Zinzani P., Ferreri A., Vitolo U., Stelitano C., Brusamolino E., Cabras G., Rigacci L., Salvi F., Gargantini L., Balzarotti M., Merli F., Pinotti G., Montoto S., Lopez-GuillermoA., Cavalli M., Giovanella L., Johnson P.W., Martelli $M$. Prognostic value of ${ }^{18}$ FDG baseline functional PET parameters in primary diffuse large B-cell lymphoma. Hematological Oncology. 2013; 31, suppl. 1: 187-188.

7. Pinnix C.C., Ng A.K., Dabaja B.S., Milgrom S.A., Gunther J.R., Fuller C.D., Smith G.L., Yebia Z.A., Qiao W., Wogan C.F., Akhtari M., Mawlawi O., Medeiros L.J., Chuang H.H., Martin-Doyle W., Armand P., LaCasce A.S., Oki Y., Fanale M., Westin J., Neelapu S., Nastoupil L. Position emission tomography-computed tomography predictors of progression after DA-R-EPOCH for PMBCL. Blood Adv. 2018; Jun 12; 2(11): 1334-1343, PMID: 29895624 , PMCID: PMCID: PMC5998935. doi: 10.1182/bloodadvances.2018017681.

8. Tavares M., Simas A.M., Santo A.E., Domingues N., Oliveira I., Moreira I., Martins A., Viterbo L., Henrique R., Chacim S., MarizJ. Favorable outcome of primary mediastinal diffuse large B-cell lymphoma: a Portugal Single-Center Experience // Hematol Oncol. 2015; 33, suppl. 1: 294-295. doi: 10.1002/hon.

9. Trofimova O.P., Zavodnova I.Z., Tumyan G.S., Pryamikova Yu.I., Volkova N.V., Zaichenko O.S., Nazarenko A.V. Modern radiotherapy in multimodal treatment of patients with primary mediastinal (thymic) B-large cell lymphoma (results of treatment of 131 patients at The N.N. Blokhin national cancer RESEARCH center of the Ministry of health of Russia). Modern oncology. 2018; 20(4): 5-15. (in Russ)

10. Vassilakopoulos T.P., Papageorgiou S.G., Michail M., Angelopoulou M.K., Kourti G., Kalpadakis C., Kotsopoulou M., Leonidopoulou T., Konstantinidou P., KotsianidisI., Boutsis D., Michali E., Sachanas S., Terpos E., Karinakis G., Poziopoulos C., Vadikolia C., Pigaditou A., Vrakidou E., Anargyrou K., Symeonidis A., Stefanoudaki E., Hadjibarissi E., Papaioannou M., Gainaru G., Tsirogianni M., Katodritou E., Karmiris T., Variami E., Pappa V., Dimopoulos M., Roussou P., Panaytidis P., Konstantopoulos K., Pangalis G.A. Prognostic factors (pfs) in primary mediastinal large b-cell lymphoma (pmlbcl) treated with rituximab-chop (rchop) \pm radiotherapy (rt). Hematological Oncology. 2017; 37, Issue S2: 266267. https://doi.org/10.1002/hon.76_2630.

11. Dunleavy K. Primary mediastinal B-cell lymphoma: biology and evolving therapeutic strategies. Hematology Am Soc Hematol Educ Program. 2017 Dec 8; 2017 (1): 298-303. PMID: 29222270 PMCID: PMC6142582. doi: 10.1182/ asheducation-2017.1.298.

12. Salek D., Janikova A., Michalka J., Miculkova E., Hadrabova M., Folber F., Kral Z., Mayer J. First-line adjuvant radiotherapy improves PFS in primary mediastinal B-cell lymphoma - a retrospective analysis of 102 patients from a single center. Hematological Oncology. 2019; 37, suppl. 2: 458. https://doi.org/10.1002/hon.134_2631.

13. Hayden A., Tonseth P., Villa D., Gerrie A.S., Scott D.W., Freeman C.L., Slack G.W., Parsons C., Pickles T., Wilson D., Connors J.M., Sebn L.H., Savage K.L. Outcome of primary mediastinal large B-cell lymphoma in the rituximab era: impact of a PET-guided approach. Hematological Oncology. 2019. 37, suppl. 2: 156-157. https://doi.org/10.1002/ hon.113_2629.

14. Trneny M., Polgarova K., Janikova A., Belada D., Prochazka V., Duras J., Mocikova H., Steinerova K., Campr V., Blabovcova P., Petrova M., Zogala D., Ptachik V. Is it radiotherapy necessary for primary mediastinal B-cell lymphoma (PMBL) patients achieving PET negativity after immunochemotherapy? Hematological Oncology. 2019. 37, suppl. 2: 267-268. https://doi.org/10.1002/hon.77_2630.

15.Jackson M.W., Rusthoven C.G.,Jones B.L., Kamdar M., Rabinovitch R. Improved survival with combined modality therapy in the modern era for primary mediastinal B-cell lymphoma. Am J Hematol. 2016 May; 91(5): 476-80. doi:10.1002/ajh.24325 PMID: 26852276.

16. Savage K.J., Al-Rajhi N., Voss N., Paltiel C., Klasa R., Gascoyne R.D., Connors J.M. Favorable outcome of primary mediastinal large B-cell lymphoma in a single institution: the British Columbia experience. Ann Oncol. 2006 Jan; 17(1): 123-30. doi: 10.1093/annonc/mdj030. PMID: 16236753.

17.Dunleavy K., Pittaluga S., Maeda L.S., Advani R., Chen C.C., Hessler J., Steinberg S.M., Grant C., Wright G., Varma G., Staudt L.M., Jaffe E.S., Wilson W.H. Dose-adjusted EPOCH-rituximab therapy in primary mediastinal B-cell lymphoma. N Engl J Med. 2013 Apr 11; 368(15): 1408-16. doi: 10.1056/NEJMoa1214561. PMID: 23574119 PMCID: PMC4568999.

18. Milani Ch., Advani R., Roschewski M., Walters K.M., Chen C.C., Baratto L., Ablman M.A., Miljkovic M.D., Steinberg S.M., Lam J., Shovlin M., Dunleavy K., Pittaluga S., Jaffe E.S., Wilson W.H. End of treatment and serial PET imaging in primary mediastinal B-cell lymphoma following dose-adjusted EPOCH-R: a paradigm shift in clinical decision making. Haematologica. 2018 Aug; 103(8): 1337-1344. doi: 10.3324/haematol.2018.192492 PMID: 29748435 PMCID: PMC6068044.

19.Aoki T., Shimada K., Suzuki R., Izutsu K., Tomita A., Maeda Y., Takizawa J., Mitani K., Igarashi T., Sakai K., Miyazaki K., Mihara K., Obmachi K., Nakamura N., Takasaki H., Kiyoi H., Nakamura S., Kinoshita T., Ogura M. High-dose chemotherapy followed by autologous stem cell transplantation for relapsed/refractory primary mediastinal large B-cell lymphoma. Blood Cancer J. 2015 Dec 4; 5(12): e372. doi: 10.1038/bcj.2015.101. PMID: 26636287 PMCID: PMC4735068.

20. Broccoli A., Casadei B., Stefoni V., Pellegrini C., Quirini F., Tonialini L., Morigi A., Marangon M., Argnani L., Zinzani P.L. The treatment of primary mediastinal large B-cell lymphoma: a two decades monocentric experience with 98 patients. BMC Cancer. 2017 Apr 17; 17(1): 276. doi: 10.1186/s12885-017-3269-6. PMID: 28415982 PMCID: PMC5392963. 
21. Chan E.L., Koh L.P., Lee J., De Mel S., Jeyasekharan A., Liu X., Tang T., Lim S.T., Tao M., Quek R., M.F.B.H. Ras, Lee Y.S., Diong C., Tan D., Kim S.J., Chee Y.L., Poon L.M. Real world experience of R-CHOP with or without consolidative radiotherapy vs DA-EPOCH-R in the first-line treatment of primary mediastinal B-cell lymphoma. Cancer Med. 2019 Aug; 8(10): 4626-4632. doi: 10.1002/cam4.2347. PMID: 31264808. PMCID: PMC6712459.

22. Held G., Thurner L., Poeschel V., Berdel Ch., Ott G., Schmidt Ch., Viardot A., Borchmann P., Shpilberg O., Nickelsen M., Federico M., Brown P., Murawski N., Trumper L.H., Schmidberger H., Ruebe Ch., Fleckenstein J., Schmitz N., Loeffler M., Ziepert M. Role of radiotherapy and dose-densification of R-CHOP in primary mediastinal B-cell lymphoma: a subgroup analysis of the unfolder trial of the German Lymphoma Alliance (GLA). J Clin Oncol. 2020 May; 38(15 suppl): 8041-8041. doi: 10.1200/JCO.2020.38.15 suppl.8041.

23. Carbone P.P., Kaplan H.S., MusshoffK., Smithers D.W., Tubiana M. Report of the Committee on Hodgkin's disease staging classification. Cancer Res. 1971 Nov; 31(11): 1860-1. PMID: 5121694.

24. Lister T.A., Crowther D., Sutcliffe S.B., Glatstein E., Canellos G.P., Young R.C., Rosenberg S.A., Coltman C.A., Tubiana M. Report of a committee convened to discuss the evaluation and staging of patients with Hodgkin's disease: Cotswolds Meeting. J Clin Oncol. 1989 Nov; 7(11): 1630-6. doi: 10.1200/JCO.1989.7.11.1630. PMID: 2809679.

25. Cheson B.D., Fisher R.I., Barrington S.F. Recommendations for initial evaluation, staging, and response assessment of Hodgkin and non-Hodgkin lymphoma: the Lugano classification. J Clin Oncol. 2014 Sep 20; 32(27): 3059-68. doi: 10.1200/JCO.2013.54.8800. PMID: 25113753 PMCID: PMC4979083.

26. Specht L., Yahalom J., Illidje T., Berthelsen A.K., Constine L.S., Eich H.T., Girinsky Th., Hoppe R.T., Mauch P., Mikbaeel N.G., Ng A. ILROG. Modern radiation therapy for Hodgkin lymphoma: field and dose guidelines from the international lymphoma radiation oncology group (ILROG). Int J Radiat Oncol Biol Phys 2014 Jul 15; 89(4): 854-62. doi: 10.1016/j.ijrobp.2013.05.005. PMID: 23790512.

27. Cheson B. D., Horning S., Coiffer B., Shipp M., Fisher R., Connors J. Report of an international workshop to standardize response criteria for non Hodgkin's lymphomas. NCI Sponsored International Working Group. J Clin Oncol. 1999 Apr; 17(4): 1244. doi: 10.1200/JCO.1999.17.4.1244. PMID: 10561185.

28. Cheson B.D., Pfistner B., Juweid M., Gascoyne R.D., Specht L., Horning S.J. Revised response criteria for malignant lymphoma. J Clin Oncol. 2007 Feb 10; 25(5): 579-86. doi: 10.1200/JCO.2006.09.2403. PMID: 17242396.

29. Cheson B.D. PET/CT in lymphoma: current overview and future direction. Semin Nucl Med. 2018 Jan; 48(1): 76-81. doi: 10.1053/j.semnuclmed.2017.09.007 PMID: 29195620. 